Bárbara Borgatta

Marcos Pérez

Loreto Vidaur

Leonardo Lorente

Lorenzo Socías

Juan Carlos Pozo

José Garnacho-Montero

Jordi Rello

The pH1N1 GTEI/SEMICYUC Investigators

\section{Erratum to: Elevation of creatine kinase is associated with worse outcomes in 2009 pH1N1 influenza A infection}

Published online: 27 June 2012

(C) Copyright jointly held by Springer and ESICM 2012

The online version of the original article can be found under doi:10.1007/s00134-012-2565-5.

B. Borgatta $(\square)$

Critical Care Department, Vall D'Hebron University Hospital, Universitat Autònoma de Barcelona (UAB),

08035 Barcelona, Spain

e-mail: borgatta@crips.es

Tel.: +34-93-2746209

Fax: +34-93-2746062

M. Pérez · J. Rello

Critical Care Department, Vall D’Hebron University Hospital,

Vall D'Hebron Research Institute (VHIR), Universitat Autònoma

de Barcelona (UAB), 08035 Barcelona, Spain

L. Vidaur

Critical Care Department, Hospital Donostia,

San Sebastián, Spain

L. Lorente

Critical Care Department, Canarias University Hospital,

Santa Cruz de Tenerife, Spain

L. Socías

Critical Care Department, Hospital Son Llàtzer,

Palma de Mallorca, Spain

\section{J. C. Pozo}

Critical Care Department, Hospital Universitario Reina Sofía, Instituto Maimonides de Investigación Biomédica Cordoba (IMIBIC), Cordoba, Spain

\section{J. C. Pozo}

CIBER de Enfermedades Hepáticas y Digestivas (CIBERehd), Barcelona, Spain

\section{J. Garnacho-Montero}

Critical Care and Emergency Clinical Unit, Virgen del Rocío

University Hospital, Seville, Spain

\section{J. Rello}

CIBER Enfermedades Respiratorias (CIBERes), Barcelona, Spain

The pH1N1 GTEI/SEMICYUC Investigators

GTEI, SEMICYUC, Madrid, Spain

\section{Erratum to: Intensive Care Med DOI 10.1007/ s00134-012-2565-5}

Owing to an oversight, the pH1N1 GTEI/SEMICYUC Investigators were not included among the authors of this article. The complete list of authors is given here. 